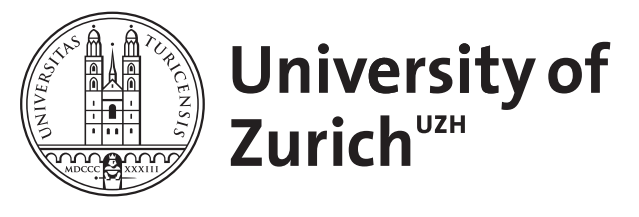

\title{
Peptide-guided assembly of repeat protein fragments
}

\author{
Michel, Erich ; Plückthun, Andreas ; Zerbe, Oliver
}

\begin{abstract}
In this communication we present the peptide-guided assembly of complementary fragments of designed armadillo repeat proteins (dArmRPs) to create proteins that bind peptides not only with high affinity but also with good selectivity. We recently demonstrated that complementary N- and C-terminal fragments of dArmRPs form high-affinity complexes that resemble the structure of the fulllength protein, and that these complexes bind their target peptides. We now demonstrate that dArmRPs can be split such that the fragments assemble only in the presence of a templating peptide, and that fragment mixtures enrich the combination with the highest affinity for this peptide. The enriched fragment combination discriminates single amino acid variations in the target peptide with high specificity. Our results suggest novel opportunities for the generation of new peptide binders by selection from dArmRP fragment mixtures.
\end{abstract}

DOI: https://doi.org/10.1002/anie.201713377

Posted at the Zurich Open Repository and Archive, University of Zurich

ZORA URL: https://doi.org/10.5167/uzh-150193

Journal Article

Accepted Version

Originally published at:

Michel, Erich; Plückthun, Andreas; Zerbe, Oliver (2018). Peptide-guided assembly of repeat protein fragments. Angewandte Chemie Internationale Edition, 57(17):4576-4579.

DOI: https://doi.org/10.1002/anie.201713377 


\title{
Peptide-Guided Assembly of Repeat Protein Fragments
}

\author{
Erich Michel ${ }^{[a] *}$, Andreas Plückthun ${ }^{[\mathrm{b}]}$ and Oliver Zerbe ${ }^{[\mathrm{a}] \star}$
}

\begin{abstract}
In this communication we present the peptide-guided assembly of complementary fragments of designed armadillo repeat proteins (dArmRPs) to create proteins that bind peptides not only with high affinity but also with good selectivity. We recently demonstrated that complementary $\mathrm{N}$ - and $\mathrm{C}$-terminal fragments of dArmRPs form high-affinity complexes that resemble the structure of the full-length protein, and that these complexes bind their target peptides. We now demonstrate that dArmRPs can be split such that the fragments assemble only in the presence of a templating peptide and that fragment mixtures enrich the combination with the highest affinity for this peptide. The enriched fragment combination discriminates single amino acid variations in the target peptide with high specificity. Our results suggest novel opportunities for the generation of new peptide binders by selection from dArmRP fragment mixtures.
\end{abstract}

Directed evolution methods have been very successfully used over the past years to create binding proteins that target peptides, proteins and even small molecules with high affinity and high selectivity $(\underline{1-3})$. These procedures have used both antibody and non-antibody scaffolds, and they aim at modifying loops and/or surfaces to become complementary to the target and thus suitable for forming sufficient intermolecular contacts while retaining the overall fold of the scaffolding protein (4-7). In this work we investigate whether the target recognition surface can also be provided by two complementary protein fragments that form a complex capable of binding the target.

dArmRPs form elongated, rod-like molecules that are comprised of multiple, tightly packed internal modules $\mathrm{M}$, and which are terminated at the $\mathrm{N}$ - and $\mathrm{C}$-terminal ends by capping modules $Y_{i i i}$ and $A_{i i}$, respectively (8-11). Each internal module $M$ contains three tightly packed alpha helices $\mathrm{H} 1, \mathrm{H} 2$, and $\mathrm{H} 3$ (Fig. 1A). They propagate a right-handed triangular spiral (Fig. 1B), which exposes a supercoiled binding surface consisting of helices $\mathrm{H} 3$. Each module specifically recognizes two amino acids of an extended target peptide (Fig. 1B,C) $(\underline{12}, \underline{13})$. dArmRPs have been shown to bind $(K R)_{n}$-type peptides within a $K_{d}$ that depends both on the numbers of $M$ modules and (KR)-dipeptide units in the peptide (13). We recently discovered that the complementary dArmRP fragments $\mathrm{YM}_{2}$ and MA assemble with

[a] Dr. Erich Michel, Prof. Oliver Zerbe Department of Chemistry University of Zurich

Winterthurerstrasse 190, CH-8057 Zurich

E-mail: erichxmichel@gmail.com; oliver.zerbe@chem.uzh.ch

[b] Prof. Andreas Plückthun

Department of Biochemistry

University of Zurich

Winterthurerstrasse 190, CH-8057 Zurich

Supporting information for this article is given via a link at the end of the document. a $\mathrm{K}_{\mathrm{d}}$ of $126 \mathrm{nM}$ into the $\mathrm{YM}_{2}: \mathrm{MA}$ complex that structurally resembles the full-length $\mathrm{YM}_{3} \mathrm{~A}$ protein (14). Importantly, the assembled fragments of a dArmRP binder for the peptide ligand neurotensin retained the ability to bind the peptide (8).
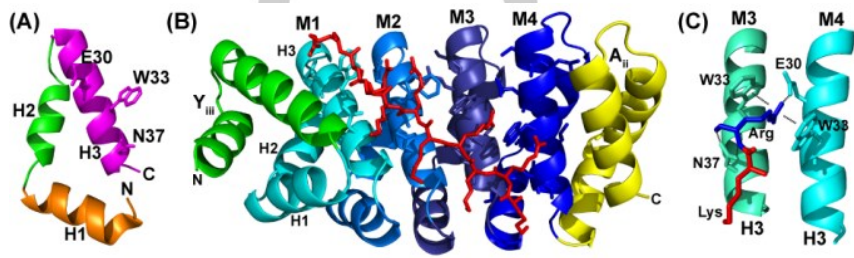

Figure 1. Structural features of designed armadillo repeat proteins. (A) dArmRPs contain internal 42-residue $M$ modules which consist of three alpha helices $\mathrm{H} 1, \mathrm{H} 2$ and $\mathrm{H} 3$. The capping modules $Y_{\mathrm{iii}}$ and $\mathrm{A}_{\mathrm{ii}}$ are comprised of 31 and 41 residues, respectively. (B) $A$ model of $\mathrm{YM}_{4} \mathrm{~A}$ based on the crystal structure of $Y M_{5} A(14)$. The bound $(K R)_{4}$ peptide is shown in red. (C) Detailed view of the modular $(K R)$ dipeptide recognition: each arginine of the peptide specifically forms salt-bridges with Glu30, $\pi$-cation interactions with Trp33, and a double $\mathrm{H}$-bond of Asn37 to the peptide backbone.

Based on these observations we set out to investigate whether mixtures of complementary dArmRP fragments enrich those combinations that constitute the best binder towards a given target peptide. The principle is demonstrated for the protein $\mathrm{YM}_{4} \mathrm{~A}$ using mixtures of a particular $\mathrm{N}$-terminal fragment with a number of complementary $\mathrm{C}$-terminal fragments that display different affinities towards a target peptide (Fig. 2).

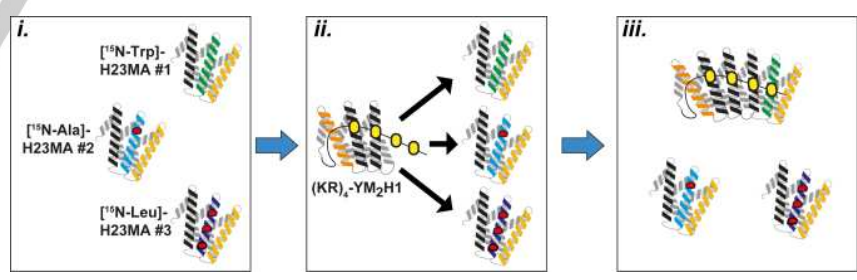

Figure 2. Peptide-guided dArmRP fragment assembly. (i) Three C-termina $\mathrm{H} 23 \mathrm{MA}$ dArmRPs fragments, in which $\mathrm{H} 23$ denotes the second and third helix of a $\mathrm{M}$ module, with different affinities to a target peptide (mutations shown in red) are (ii) mixed with $(\mathrm{KR})_{4}-\mathrm{YM}_{2} \mathrm{H} 1$, which is a fusion of the $(\mathrm{KR})_{4}$ target peptide with the complementary $\mathrm{N}$-terminal $\mathrm{YM}_{2} \mathrm{H} 1$ fragment $(\mathrm{H} 1$ here is the first helix of a $\mathrm{M}$ module). (iii) $(\mathrm{KR})_{4}-\mathrm{YM}_{2} \mathrm{H} 1$ enriches the C-terminal H23MA fragment that has the highest binding affinity to the target peptide to form a complex (top) over more weakly binding fragments (bottom). Modules that are kept constant throughout the selection procedure are shown in black while the green, light blue or dark blue modules represent the variable module.

Our initial setup comprised $\mathrm{YM}_{2}$ and $\mathrm{M}_{2} \mathrm{~A}$ fragments obtained from an inter-modular split of the $\mathrm{YM}_{4} \mathrm{~A}$ dArmRP. However, the high-affinity interaction of complementary fragments from the inter-modular split, even in the absence of ligand, caused an enrichment bias to fragments with highest affinity for the respective complementary partner fragment, independent of peptide affinity. This greatly reduced the impact of the ligand to 
effectively guide assembly of those fragment combinations that show highest affinity to the ligand (data not shown). We therefore aimed for alternative fragmentation sites which avoid fragment assembly in the absence of the templating peptide altogether. We thus introduced intra-modular split sites between helices $\mathrm{H} 1$ and $\mathrm{H} 2$ and between helices $\mathrm{H}_{2}$ and $\mathrm{H} 3$ (Fig. 3A) and determined the complementation affinities of the corresponding pairs by analytical size exclusion chromatography (Fig. 3B-E).
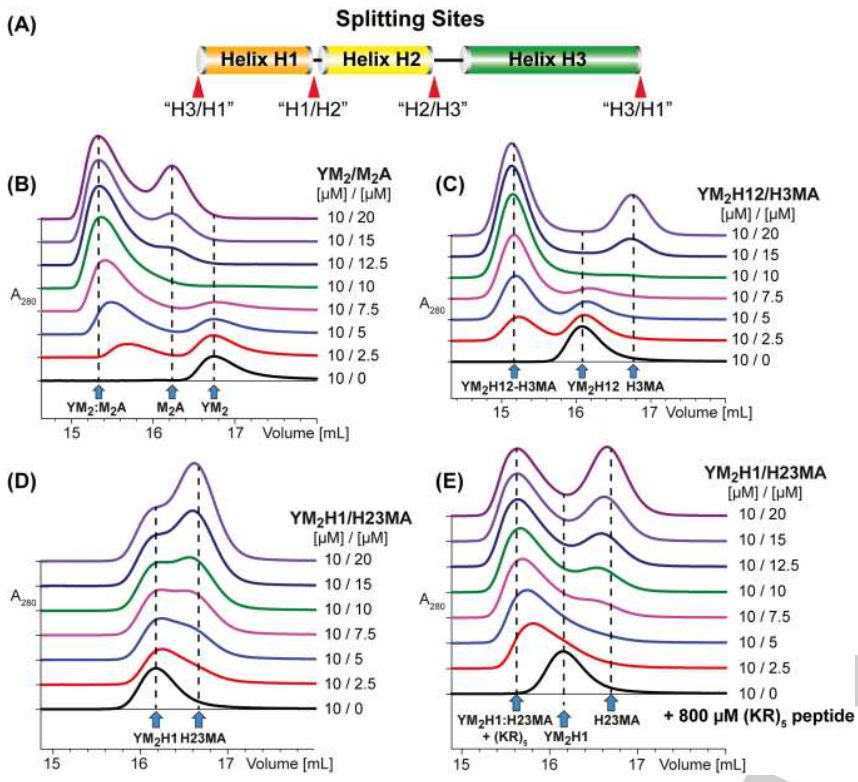

Figure 3. Complementation properties of split dArmRPs. (A) Location of the analyzed split sites. (B-E) Size-exclusion profiles of the various N-terminal fragments titrated with increasing amounts of the complementary $\mathrm{C}$-terminal fragments: (B) $\mathrm{YM}_{2}: \mathrm{M}_{2} \mathrm{~A}(\mathrm{H} 3: \mathrm{H} 1)$, (C) $\mathrm{YM}_{2} \mathrm{H} 12: \mathrm{H} 3 \mathrm{MA}$, (D) $\mathrm{YM}_{2} \mathrm{H} 1: \mathrm{H} 23 \mathrm{MA}$, and (E) $\mathrm{YM}_{2} \mathrm{H} 1: \mathrm{M} 23 \mathrm{MA}$ in presence of 80 equiv. of $(\mathrm{KR})_{5}$. The concentrations of $\mathrm{N}$ and $\mathrm{C}$-terminal fragments are indicated next to each elution profile.

The stepwise addition of $M_{2} A$ to $Y M_{2}$ results in immediate and stoichiometric complementation, evident from the $\mathrm{YM}_{2}: \mathrm{M}_{2} A$ peak eluting at earlier volumes (Fig. 3B). The $\mathrm{YM}_{2} \mathrm{H} 12$ and $\mathrm{H} 3 \mathrm{MA}$ pair displays a similar SEC behavior, indicating a comparable affinity in complementation (Fig. 3C). In contrast, the absence of a peak at earlier elution volumes clearly indicates that $\mathrm{YM}_{2} \mathrm{H} 1$ and H23MA do not complement each other in the absence of peptide (Fig. 3D). Subsequent isothermal titration calorimetry (ITC) measurements indicated a $K_{d}$ of $593 \pm 35 \mathrm{nM}$ for assembly of the $\mathrm{YM}_{2}: \mathrm{M}_{2} \mathrm{~A}$ pair, a $\mathrm{K}_{\mathrm{d}}$ of $234 \pm 62 \mathrm{nM}$ for the $\mathrm{YM}_{2} \mathrm{H} 12: \mathrm{H} 3 \mathrm{MA}$ pair and $a K_{d}$ of $>20 \mu \mathrm{M}$ for the $\mathrm{YM}_{2} \mathrm{H} 1$ :H23MA pair. However, when carrying out the SEC experiment with the $\mathrm{YM}_{2} \mathrm{H} 1: \mathrm{H} 23 \mathrm{MA}$ pair in the presence of excess $(\mathrm{KR})_{5}$ peptide (80 equiv.), we observed stoichiometric fragment complementation (Fig. 3E). The $\mathrm{YM}_{2} \mathrm{H} 1 \mathrm{H}$ : $23 \mathrm{MA}$ pair therefore represents the desired conditional complementation system that strictly requires the presence of the target peptide.

To facilitate the NMR analysis later on, we converted the trimolecular into a bi-molecular complex by fusing $(\mathrm{KR})_{4}$ via a $(\mathrm{GS})_{\mathrm{n}}$ linker to the $\mathrm{N}$-terminal $\mathrm{YM}_{2} \mathrm{H} 1$ fragment. This system maintains the conditional assembly, as only C-terminal fragments with a significant interaction with the (KR)-peptide assemble with the $\mathrm{N}$-terminal fragment. ITC analysis revealed that the $(\mathrm{KR})_{4}-(\mathrm{GS})_{4} \mathrm{G}-\mathrm{YM}_{2} \mathrm{H} 1$ fusion binds $\mathrm{H} 23 \mathrm{MA}$ with an $\mathrm{K}_{\mathrm{d}}$ of $18 \mathrm{nM}$ (Table 1), which is roughly three orders of magnitude better than the $\mathrm{YM}_{2} \mathrm{H} 1: \mathrm{H} 23 \mathrm{MA}$ complementation in the absence of ligand. Systematic variation of the linker length between the peptide and the $\mathrm{YM}_{2} \mathrm{H} 1$ domain indicates a minimum of 9 alternating Gly and Ser residues, $(\mathrm{GS})_{4} \mathrm{G}$, for an optimal interaction and shows a dramatic reduction of the affinity when the linker is too short $\left(\mathrm{K}_{d}\right.$ ca. $1.5 \mu \mathrm{M}$ with 8 residues and $>8 \mu \mathrm{M}$ with 7 linker residues). We therefore continued with the (GS) ${ }_{4} G$ linker construct, which in the following is referred to as $(K R)_{4}$ $\mathrm{YM}_{2} \mathrm{H} 1$.

The design of H23MA variants with reduced affinities towards $(K R)_{n}$ peptides was inspired by the crystal structure of $\mathrm{YM}_{5} \mathrm{~A}$ bound to $(K R)_{5}$ that revealed specific and modular recognition of each arginine through electrostatic interactions with Glu30 and $\pi$-cation interactions with Trp33, as well as by the peptide bond forming $\mathrm{H}$-bonds to the conserved Asn37 (Fig. 1C) (13). Hence various residues were replaced by alanine in the most $\mathrm{C}$ terminal $\mathrm{M}$ module so that the binding interface to the $\mathrm{N}$-terminal fragment remained unchanged. This resulted in variants [2-W]H23MA with mutation in Trp37, and [2-EWN]-H23MA where Glu30, Trp33 and Asn37 simultaneously were mutated to Ala. ITC analysis of their interaction with $(\mathrm{KR})_{4}-\mathrm{YM}_{2} \mathrm{H} 1$ revealed a $\mathrm{K}_{\mathrm{d}}$ of $33 \mathrm{nM}$ for [2-W]-H23MA and a $\mathrm{K}_{d}$ of $137 \mathrm{nM}$ for [2-EWN]$\mathrm{H} 23 \mathrm{MA}$, which can be compared to a $\mathrm{K}_{\mathrm{d}}$ of $18 \mathrm{nM}$ for interaction with the wild-type H23MA (Table 1).

Table 1: Dissociation constants and $\Delta \Delta G$ for binding of $(K R)_{4}$ target peptide and dArmRP mutants

\begin{tabular}{llllll}
\hline No & $\begin{array}{l}\text { XXXX- } \\
\text { KRKR* }\end{array}$ & H23MA & $\begin{array}{l}\mathrm{K}_{\mathrm{d}} \\
{[\mathrm{nM}]}\end{array}$ & $\begin{array}{l}\Delta \Delta \mathrm{G} \text { to wt } \\
{[\mathrm{kJ} / \mathrm{mol}]}\end{array}$ & $\begin{array}{l}\Delta \Delta \mathrm{G} \text { (calc.) } \\
{[\mathrm{kJ} / \mathrm{mol}]}\end{array}$ \\
\hline 1 & wt & wt & $17.5 \pm 0.1$ & 0 & - \\
$\underline{2}$ & $\underline{\text { ARKR }}$ & wt & $24.9 \pm 3.3$ & $0.9 \pm 0.3$ & - \\
3 & KRAA & wt & $248 \pm 25$ & $6.6 \pm 0.2$ & - \\
$\underline{4}$ & $\underline{\text { ARAA }}$ & wt & $434 \pm 32$ & $8.0 \pm 0.2$ & 7.4 (Nos. 2+3) \\
$\underline{5}$ & $\underline{\text { AAAA }}$ & wt & $3890 \pm 117$ & $13.4 \pm 0.1$ & - \\
$\underline{6}$ & $\underline{\text { AEAA }}$ & wt & $6070 \pm 417$ & $14.5 \pm 0.2$ & - \\
7 & wt & $2-\mathrm{W}$ & $32.9 \pm 28.4$ & $1.6 \pm 1.8$ & - \\
8 & wt & $2-E N$ & $84.7 \pm 8.6$ & 3.9 & - \\
9 & wt & 2-EWN & $137 \pm 38$ & $5.0 \pm 0.7$ & 5.5 (Nos. 7+8) \\
$\underline{10}$ & $\underline{\text { ARAA }}$ & 2-W & $971 \pm 13$ & $10.0 \pm 0.1$ & 9.5 (Nos. 4+7) \\
$\underline{11}$ & $\underline{\text { ARAA }}$ & 2-EN & $2340 \pm 54$ & $12.1 \pm 0.1$ & 11.9 (Nos. 4+8) \\
$\underline{12}$ & $\underline{\text { ARAA }}$ & 2-EWN & $5090 \pm 707$ & $14.0 \pm 0.4$ & 13.4 (Nos. 4+9)
\end{tabular}

*shown are only sequences of the first four residues, wt $=\mathrm{KRKR}$

To also investigate the selectivity of the enriched fragment combination for different target peptide sequences, we prepared $(\mathrm{KR})_{4}-\mathrm{YM}_{2} \mathrm{H} 1$ variants that contained mutations in the $\mathrm{N}$-terminal tetrapeptide KRKR that interacts with the C-terminal H23MA fragment. To isolate the influence of a single target residue, we replaced three target residues by Alanines and altered the residue of interest that faces the Arg binding pocket of the terminal $M$ module in H23MA. ITC analysis of wt-H23MA with three (AXAAKRKR)- $\mathrm{YM}_{2} \mathrm{H} 1$ constructs, with $\mathrm{X}$ being either $\mathrm{Arg}$, 
Ala, or Glu, revealed $K_{d}$ values of $0.43 \mu \mathrm{M}, 3.89 \mu \mathrm{M}$, and 6.07 $\mu \mathrm{M}$, respectively (Table 1 ). The clear preferential binding to the Arg variant confirms the high selectivity in our split dArmRP fragment complementation system. Interestingly, (AEAAKRKR)$\mathrm{YM}_{2} \mathrm{H} 1$ binds even more weakly to H23MA compared to the Ala variant, which is likely due to an electrostatic repulsion between Glu in the peptide with Glu37 of the Arginine pocket (Fig.1).

Using the $K_{d}$ values obtained from the ITC analysis of the $(K R)_{4^{-}}$ $\mathrm{YM}_{2} \mathrm{H} 1$ variants with wt-H23MA and the [2-W]-, [2-EN]-, and [2EWN]-H23MA mutants, we calculated the Gibbs free energy differences, $\Delta \Delta \mathrm{G}$, to the wild-type $(\mathrm{KR})_{4}-\mathrm{YM}_{2} \mathrm{H} 1: \mathrm{H} 23 \mathrm{MA}$ interaction. We observed that changes in $\Delta \Delta G$ due to multiple mutations are essentially additive (Table 1 ). The perturbation of specific interactions between the $\mathrm{M}$ module and the Arg residue of the target peptide is additive, in a first approximation, irrespective of whether the mutations occur in the M module ([2EWN]-H23MA) or in the peptide ((AAAAKRKR)-YM ${ }_{2} \mathrm{H} 1$; Table 2). This feature illustrates the robustness of target specificity, allowing to alter single residues without triggering large and unpredictable structural changes in the dArmRP.

A clear advantage of the conditional assembly is the absence of a complementation bias in the absence of peptide, and that discrimination between C-terminal fragments is solely dictated by the relative energetic contribution of the arginine binding to the terminal $\mathrm{M}$ module in the H23MA variants. Nonetheless, the affinity of $(\mathrm{KR})_{4}-\mathrm{YM}_{2} \mathrm{H} 1$ to the three C-terminal fragments wtH23MA, [2-W]-H23MA and [2-EWN]-H23MA, with $K_{d}$ 's of $18 \mathrm{nM}$, $33 \mathrm{nM}$ and $137 \mathrm{nM}$ are relatively similar, initially raising the question whether the C-terminal wt-fragment can still be enriched in the mixture. We thus aimed at using NMR to analyze the populations in an equimolar mixture of $(\mathrm{KR})_{4}-\mathrm{YM}_{2} \mathrm{H} 1$ and the three C-terminal fragments wt-H23MA, [2-W]-H23MA and [2EWN]-H23MA. To this end we first prepared uniformly $\left[{ }^{13} \mathrm{C},{ }^{15} \mathrm{~N}\right]-$ labeled H23MA and assigned the protein backbone resonances. Secondary chemical shifts $(\underline{15})$ and ${ }^{15} \mathrm{~N}\left\{{ }^{1} \mathrm{H}\right\}-\mathrm{NOE}$ data revealed rigid helix formation in helices $\mathrm{H} 2$ and $\mathrm{H} 3$ of the first truncated module, as well as in the second module and the C-cap (Fig. S1, S2). A subsequent NMR titration of uniformly $\left[{ }^{15} \mathrm{~N}\right]$-labeled $\mathrm{H} 23 \mathrm{MA}$ with an excess of unlabeled $\mathrm{N}$-terminal $\mathrm{YM}_{2} \mathrm{H} 1$ revealed only small chemical shift perturbations (CSP) in the fast exchange regime (Fig. S3), indicative of a $K_{d}$ in the $\mu \mathrm{M}$ to $\mathrm{mM}$ range, which confirms our ITC measurements. In contrast, the addition of the peptide fusion $(\mathrm{KR})_{4}-\mathrm{YM}_{2} \mathrm{H} 1$ to uniformly $\left[{ }^{15} \mathrm{~N}\right]-$ labeled H23MA resulted in large CSPs in the slow exchange regime, which suggests a $K_{d}$ in the $n M$ range. This slow exchange allows one to relate the disappearance of resonances corresponding to the free state to the formation of the assembled state. Integration of resonances from residues sensitive to complementation for all three C-terminal variants therefore quantifies free and thus bound states of these constructs in the mixture.

To be able to discriminate and quantify the populations of each C-terminal H23MA fragment variant we $\left[{ }^{15} \mathrm{~N}\right]$-labeled each of them with a different amino acid type. We chose Ala, Trp and Leu residues, because many of these residues form different contacts in the free and assembled states.

\begin{tabular}{|c|c|c|c|c|}
\hline \multicolumn{4}{|c|}{ Added equivalents $(\mathrm{KR})_{4}-\mathrm{YM}_{2} \mathrm{H} 1$} & \multirow{2}{*}{$\begin{array}{l}\omega_{1}\left({ }^{15} \mathrm{~N}\right) \\
{[\mathrm{ppm}]}\end{array}$} \\
\hline 0 & 0.5 & 1 & 1.5 & \\
\hline $\begin{array}{l}\text { W149 • @ } \\
\text { W191 }\end{array}$ & $\begin{array}{l}\text { W149 } \\
\text { W191 }\end{array}$ & $\begin{array}{l}\text { W149 } \\
\text { W191 }\end{array}$ & $\begin{array}{l}\text { W149 } \\
\text { W191 }\end{array}$ & $\begin{array}{l}-121 \\
-122\end{array}$ \\
\hline 8.6 & 8.6 & 8.6 & 8.6 & \\
\hline $\begin{array}{l}\text { W149-SC } \\
8 \text { W191-SC }\end{array}$ & $\begin{array}{l}\text { W149-SC } \\
\text { W191-SC }\end{array}$ & \begin{tabular}{|l|} 
W149-SC \\
W191-SC
\end{tabular} & $\begin{array}{r}\text { W149-SC } \\
\text { W191-SC }\end{array}$ & $\begin{array}{l}-128 \\
-129\end{array}$ \\
\hline $10 \quad 9.6$ & 9.6 & 10 & 10 & \\
\hline 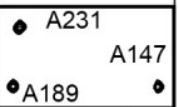 & 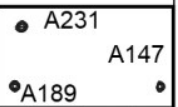 & 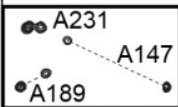 & $\begin{array}{l}A 231 \\
0\end{array}$ & -124 \\
\hline 8.8 & $8.8 \quad 8.4$ & $8.8 \quad 8.4$ & $8.8 \quad 8.4$ & \\
\hline $\begin{aligned} & \text { L136. } \\
& \bullet \\
& \text { L178 } \\
&\end{aligned}$ & \begin{tabular}{rr|} 
L136. & \\
& $\bullet$ \\
& $\bullet$ L178 \\
\end{tabular} & $\mathrm{L}^{\circ}$ & L136。 & -115 \\
\hline 7.2 & 7.2 & $\omega_{2}\left({ }^{1} \mathrm{H}\right)[\mathrm{ppm}]$ & $7.6 \quad 7.2$ & \\
\hline
\end{tabular}

Figure 4. Population analysis in the $(\mathrm{KR})_{4}-\mathrm{YM}_{2} \mathrm{H} 1: \mathrm{H} 23 \mathrm{MA}$ mixture Expansions of $\left[{ }^{15} \mathrm{~N},{ }^{1} \mathrm{H}\right]-\mathrm{HSQC}$ spectra of a mixture containing $18 \mathrm{nmol}$ of each $\left[{ }^{15} \mathrm{~N}-\mathrm{Trp}\right]-\mathrm{wt}-\mathrm{H} 23 \mathrm{MA},\left[{ }^{15} \mathrm{~N}-\mathrm{Ala}\right]-[2 \mathrm{~W}]-\mathrm{H} 23 \mathrm{MA}$ and $\left[{ }^{15} \mathrm{~N}-\mathrm{Leu}\right]-[2 \mathrm{EWN}]-\mathrm{H} 23 \mathrm{MA}$ in the presence of increasing amounts of unlabeled $(\mathrm{KR})_{4}-\mathrm{YM}_{2} \mathrm{H} 1$ are shown for selected regions that display characteristic peaks corresponding to the free and assembled states of H23MA fragment variants. SC denotes the Trp indole side chain resonance.

Our home-made $E$. coli-based cell-free expression system (16, 17) was used to prepare the $\left[{ }^{15} \mathrm{~N}-\mathrm{Trp}\right]$-labeled wt-H23MA, $\left[{ }^{15} \mathrm{~N}-\right.$ Ala]-labeled [2-W]-H23MA and $\left[{ }^{15} \mathrm{~N}\right.$-Leu]-labeled [2-EWN]H23MA constructs. Comparison of $\left[{ }^{15} \mathrm{~N},{ }^{1} \mathrm{H}\right]-\mathrm{HSQC}$ spectra of the individual H23MA variants with a spectrum of an equimolar mixture indicates that the $\mathrm{C}$-terminal fragments do not interact with each other in the mixture (Fig. S4).

Stepwise addition of $(\mathrm{KR})_{4}-\mathrm{YM}_{2} \mathrm{H} 1$ to an equimolar mixture of the three amino acid-selective ${ }^{15} \mathrm{~N}$ labeled $\mathrm{H} 23 \mathrm{MA}$ variants revealed preferential binding of the $\mathrm{N}$-terminal fragment to wt-H23MA (Fig. 4, Table 2). For example, the indole resonance of Trp-149, which stems from wild-type H23MA, is affected by addition of $(\mathrm{KR})_{4}-\mathrm{YM}_{2} \mathrm{H} 1$ while the amide moiety from Leu-136, that is part of the triple mutant, is not affected (Fig. 4). This enrichment is particularly pronounced in the presence of only 0.5 equivalents of $(\mathrm{KR})_{4}-\mathrm{YM}_{2} \mathrm{H} 1$, which interacts with ca. $30 \%$ wt-H23MA, $18 \%$ [2-W]-H23MA and only $2 \%$ [2-EWN]-H23MA. This experiment clearly demonstrates the successful enrichment of the best fragment combination from a mixture, where only the interaction between a single $M$ module and one arginine residue of the target peptide is modulated. Therefore, the particular binding energy of a single interaction with one target residue significantly favors one dArmRP fragment over the others, even for a comparably narrow $\mathrm{K}_{d}$ range of $18-137 \mathrm{nM}$.

Table 2. Populations of $(\mathrm{KR})_{4}-\mathrm{YM}_{2} \mathrm{H} 1: \mathrm{H} 23 \mathrm{MA}$ complexes $^{\text {la }}$

\begin{tabular}{llll}
\hline $\begin{array}{l}\text { Equivalents } \\
(\mathrm{KR})_{4}-\mathrm{YM}_{2} \mathrm{H} 1\end{array}$ & \multicolumn{3}{c}{ Bound Population [\%] } \\
& wt-H23MA & {$[2 \mathrm{~W}]-\mathrm{H} 23 \mathrm{MA}$} & {$[2 \mathrm{EWN}]-\mathrm{H} 23 \mathrm{MA}$} \\
\hline 0.5 & $29.6 \pm 1.4$ & $18.4 \pm 5.5$ & $2.0 \pm 1.2$ \\
1.0 & $53.6 \pm 2.5$ & $42.2 \pm 2.2$ & $4.2 \pm 2.9$ \\
1.5 & $73.7 \pm 0.8$ & $63.5 \pm 2.5$ & $12.8 \pm 2.2$ \\
\hline
\end{tabular}


Our work has demonstrated that formation of high-affinity complexes requires the interaction of only four amino acids of the peptide with their respective binding pockets in the $\mathrm{C}$ terminal fragment - usually the binding of a tetrapeptide to dArmRP would result in complexes with very weak affinity (13). However, here the "locking in" of the side chains of the fitting peptide into the binding pockets also brings both fragments into vicinity, thereby locking the protein binding interfaces into place, releasing additional free energy due to formation of the proteinprotein complex (Fig. 5). Thus, the binding process can be considered to show cooperativity when compared to the binding of a tetrapeptide to an un-split protein. This format leads therefore to a very strong selection power, since discrimination is effectively made against a fraction of the target peptide (in our case a tetrapeptide as opposed to the entire octapeptide in an unsplit system), making it possible that the nature of a single residue has decisive discriminatory power.

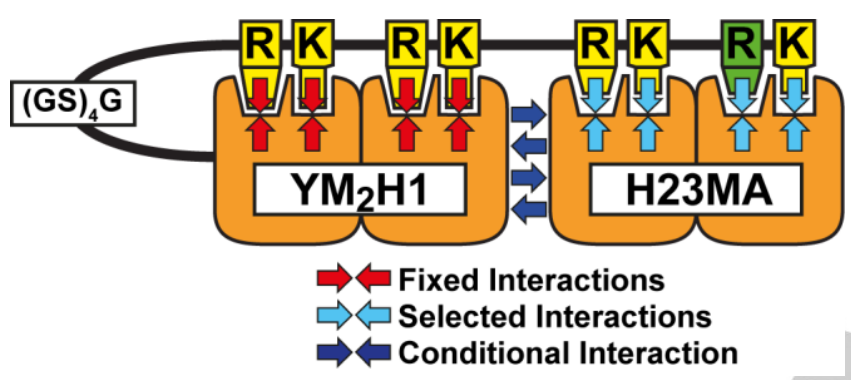

Figure 5. Schematic representation of important interactions for the conditional assembly of the (KR) ${ }_{4}-\mathrm{YM}_{2} \mathrm{H} 1: \mathrm{H} 23 \mathrm{MA}$ complex. Fixed intramolecular peptide-protein interactions that occur in the free $\mathrm{N}$-terminal fragment are shown with red arrows while cyan arrows indicate critical interactions that are required for the conditional assembly of the complementary dArmRP fragments. Additional binding energy is derived from the assembly of the complementary fragments (dark blue arrows) and strongly contributes to the overall stability of the complex. $\mathrm{N}$ - and $\mathrm{C}$-terminal caps are omitted for clarity.

Optimization of selectivity is often much more difficult to achieve than optimization for affinity. We believe that this setup will be particularly useful to optimize selectivity of binders to peptides of only slightly different sequence, e.g. when optimizing binding a single $\mathrm{M}$ module for a specific dipeptide. The extension of our mixture setup to large libraries will necessitate a coupling strategy of phenotype and genotype to unambiguously identify the amino acid sequence of the enriched binders. This could be achieved by application of commonly used display technologies, such as, e.g., ribosome-, CIS- or phage-display $(3,18,19)$. Besides selection for best binders, the presented fragmentbased recognition system could find promising applications in the development of in vivo sensor proteins, and in the formation of well-defined macromolecular assemblies in a protein-origami type fashion (20).

\section{Acknowledgements}

We acknowledge financial support for E.M. by a SINERGIA grant from the Swiss National Science Foundation (grant no. 122686) and from the Research Council of the University of
Zurich (grant no. K-73539-01-01). We thank Stefan Vetterli for help with peptide synthesis and Ilian Jelesarov for help with ITC experiments, respectively. We acknowledge Randall Watson for helpful discussions at the early stage of the project.

Keywords: repeat proteins - protein design - Armadillo repeat • split proteins $\bullet$ peptide binding $\bullet$ binding discrimination

1. Packer MS \& Liu DR (2015) Methods for the directed evolution of proteins. Nat Rev Genet 16(7):379-394.

2. Josephson K, Ricardo A, \& Szostak JW (2014) mRNA display: from basic principles to macrocycle drug discovery. Drug Discov Today 19(4):388-399.

3. Hanes J \& Plückthun A (1997) In vitro selection and evolution of functional proteins by using ribosome display. Proc Natl Acad Sci U S A 94(10):4937-4942.

4. Kuriyan J \& Cowburn D (1997) Modular peptide recognition domains in eukaryotic signaling. Annu Rev Biophys Biomol Struct 26:259-288.

5. McCafferty J \& Schofield D (2015) Identification of optimal protein binders through the use of large genetically encoded display libraries. Curr Opin Chem Biol 26:16-24.

6. Gebauer M \& Skerra A (2009) Engineered protein scaffolds as nextgeneration antibody therapeutics. Curr Opin Chem Biol 13(3):245255.

7. Plückthun A (2015) Designed ankyrin repeat proteins (DARPins) binding proteins for research, diagnostics, and therapy. Annu Rev Pharmacol Toxicol 55:489-511.

Reichen C, et al. (2016) Computationally Designed Armadillo Repeat Proteins for Modular Peptide Recognition. J Mol Biol 428(22):44674489.

9. Conti E, Uy M, Leighton L, Blobel G, \& Kuriyan J (1998)

Crystallographic analysis of the recognition of a nuclear localization signal by the nuclear import factor karyopherin alpha. Cell 94(2):193204.

10. Parmeggiani F, et al. (2008) Designed armadillo repeat proteins as general peptide-binding scaffolds: consensus design and computational optimization of the hydrophobic core. $\mathrm{J} \mathrm{Mol} \mathrm{Biol}$ 376(5):1282-1304.

11. Alfarano P, et al. (2012) Optimization of designed armadillo repeat proteins by molecular dynamics simulations and NMR spectroscopy. Protein Sci 21(9):1298-1314.

12. Reichen C, Hansen S, \& Plückthun A (2014) Modular peptide binding: from a comparison of natural binders to designed armadillo repeat proteins J Struct Biol 185(2):147-162.

Hansen S, et al. (2016) Structure and Energetic Contributions of a Designed Modular Peptide-Binding Protein with Picomolar Affinity. $J$ Am Chem Soc 138(10):3526-3532.

14. Watson RP, et al. (2014) Spontaneous self-assembly of engineered armadillo repeat protein fragments into a folded structure. Structure 22(7):985-995.

15. Wishart DS \& Sykes BD (1994) The 13C chemical-shift index: a simple method for the identification of protein secondary structure using 13C chemical-shift data. J Biomol NMR 4(2):171-180.

16. Michel E \& Wüthrich K (2012) High-yield Escherichia coli-based cellfree expression of human proteins. J Biomol NMR 53(1):43-51.

17. Michel E \& Wüthrich K (2012) Cell-free expression of disulfidecontaining eukaryotic proteins for structural biology. FEBS $J$ 279(17):3176-3184

18. McCafferty J, Griffiths AD, Winter G, \& Chiswell DJ (1990) Phage antibodies: filamentous phage displaying antibody variable domains. Nature 348(6301):552-554.

19. Odegrip R, et al. (2004) CIS display: In vitro selection of peptides from libraries of protein-DNA complexes. Proc Natl Acad Sci U S A 101(9):2806-2810.

20. Luo Q, Hou C, Bai Y, Wang R, \& Liu J (2016) Protein Assembly: Versatile Approaches to Construct Highly Ordered Nanostructures. Chem Rev 116(22):13571-13632. 
Entry for the Table of Contents (Please choose one layout)

\section{COMMUNICATION}

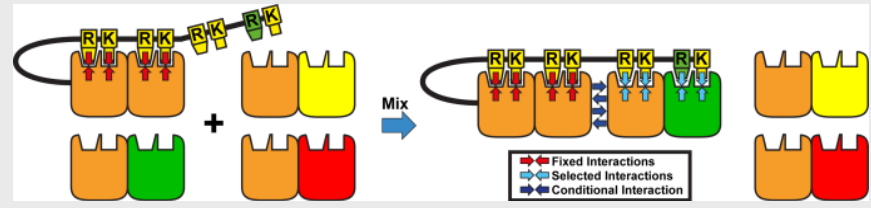

Optimizing selectivity of proteins that already bind with very good affinity to peptides

Erich Michel*, Andreas Plückthun and Oliver Zerbe*

Page No. - Page No.

Title is difficult to achieve because the selection pressure is comparably low. Here we suggest a novel method based on using complementary fragments of Armadillo repeat proteins. Using NMR we demonstrate high discriminatory power of the system. 